\title{
Is HOMA-IR a potential screening test for non-alcoholic fatty liver disease in adults with type 2 diabetes?
}

Farag allah RE ${ }^{(1)}$, Mashahit MA ${ }^{(2)}$, Hamad AA ${ }^{(3)}$ and Zaki OMA $^{(4)}$

(1) Internal medicine department, Faculty of Medicine, Fayoum University

(2) Prof of internal medicine, head of internal medicine department, Faculty of Medicine, Fayoum University

(3) lecturer of internal medicine, Faculty of Medicine, Fayoum University

(4) lecturer of clinical pathology, Faculty of Medicine, Fayoum University

Corresponding author: Remon E. Farag allah

Email address: rimonemad0@gmail.com

Tel: 01221716922

\begin{abstract}
Non-alcoholic fatty liver disease (NAFLD) is increasingly recognized as a major cause of liver related morbidity and mortality, because of its potential to progress to cirrhosis and liver failure. Patients with type 2 diabetes mellitus (T2DM) appear to have an increased risk of developing NAFLD. This study is a retrospective cohort study included 100 patients with type 2 DM from the outpatient clinic of internal medicine department at Fayoum University Hospital from (December, 2017 to June, 2018). They

were divided into two equal groups according to the presence of NAFLD by ultrasound. All patients were subjected to history taking, clinical examination and
\end{abstract}

investigations which included: ALT, AST, total cholesterol, TG, FBG and fasting insulin level.

HOMA-IR was calculated by the following formula: HOMA-IR = [plasma glucose $(\mathrm{mg} / \mathrm{dL}) \times$ plasma insulin $(\mu \mathrm{U} / \mathrm{mL})] / 405$. An association between HOMA-IR and NAFLD was found (OR: 1.25; 95\% CI: 1.04 to $1.51 ; p=0.020)$. A value of HOMA-IR of 4.2 was estimated to be an optimal threshold for discriminating NAFLD from nonNAFLD cases. 


\section{Keywords}

NAFLD; diabetes; insulin resistance.

\section{Introduction}

Non-alcoholic fatty liver disease (NAFLD) is the commonest hepatic disease in many parts of the World. [1]

Following the consensus clinical guideline of the American Association for the Study of Liver Diseases, American College of Gastroenterology, and the American Gastroenterological Association, NAFLD was defined as the presence of hepatic steatosis by imaging [ultrasonography (US) or computed tomography scanning (CT)] with no causes for secondary hepatic fat accumulation. [2]

The most credited explanation for the growing prevalence of NAFLD is the rise in such insulin-resistance conditions as obesity, the metabolic syndrome, and diabetes. Excess adiposity is associated with increased lipid influx into the liver and de novo hepatic lipogenesis, which promote hepatic triglyceride accumulation. Recently, it has been shown that the excessive rate of chronic liver disease in type 2 diabetes is similar to that of cardiovascular complications, suggesting that end-stage liver disease should be added to the list of known complications of diabetes. The prevalence of NAFLD in type 2 diabetes is
$66 \%$, double the $20 \%$ to $30 \%$ in the general population. [3]

Since the prevalence of T2DM has risen dramatically in recent years, NAFLD represents a major potential threat to public health. Systematic screening for NAFLD in adults is currently not universally recommended in primary care or among high-risk patients attending diabetes or obesity clinics, in part due to uncertainties surrounding its diagnosis and treatment, but also because a cost-effective screening test for the condition has not been established. Invasive or expensive diagnostic options, such as liver biopsy or imaging tests, should be utilised in the context of available resources, with consideration of the burden on healthcare systems and the limitation of current effective treatments for NAFLD. [4]

Insulin resistance (IR) is defined as a reduced biological response to the actions of insulin, causing the fat, muscle and liver tissues to become unable to metabolize glucose and fatty acids, being exacerbated by obesity. Thus, the association between IR and deposition of triglycerides in the liver, being evaluated by evaluation model index or the homeostasis 
model assessment-IR (HOMA-IR) is an important instrument. [5]

The Homeostasis Model Assessment of IR (HOMAIR) has proved to be a robust tool for the assessment of IR. It is determined using the following simplified equations:

\section{Patients and Methods}

This was a retrospective cohort study included 100 patients with type 2 DM from the outpatient clinic of internal medicine department at Fayoum University Hospital from (December, 2017 to June, 2018).

All the study patients were classified (according to liver ultrasound) as follows:

1. Group A.; fifty patients with NAFLD.

2. Group B.; fifty patients with normal liver.

\subsection{Inclusion criteria:}

Adult patients with type 2 diabetes mellitus.
HOMA-IR $=$ [plasma glucose $(\mathrm{mg} / \mathrm{dL}) \times$ plasma insulin $(\mu \mathrm{U} / \mathrm{mL})] / 405$.

The aim of this study is to evaluate the role of HOMA-IR in diagnosis of NAFLD in patients with type 2

DM.

\subsection{Exclusion criteria:}

Patients with any chronic liver disease other than NAFLD.

\subsection{All patients were subjected to:}

1- $\quad$ History and clinical examination.

2- $\quad$ Fasting blood sugar and fasting insulin level.

3- Total cholesterol and TGs levels.

4- $\quad$ ALT \& AST.

5- $\quad$ Abdominal US.

6- HOMA-IR will be calculated by the following formula:

HOMA-IR = [plasma glucose $(\mathrm{mg} / \mathrm{dL}) \mathrm{x}$ plasma insulin $(\mu \mathrm{U} / \mathrm{mL})]$ / 405.

\section{Results}

1- This study revealed that there was statistically significant difference regarding mean weight $(\mathrm{P}=<0.0001), \mathrm{BMI}(\mathrm{P}=<0.0001)$ and waist circumference $(\mathrm{P}=<0.0001)$ among NAFLD and control group (table 1). 
Table (1): comparison of anthropometric measurements in study groups

\begin{tabular}{|c|c|c|c|}
\hline \multirow[t]{2}{*}{$\overline{\text { Variable }}$} & $\begin{array}{l}\text { NAFLD group } \\
(\mathrm{N}=50)\end{array}$ & $\begin{array}{l}\text { Control group } \\
(\mathrm{N}=50)\end{array}$ & \multirow[t]{2}{*}{$\overline{\text { P-value }}$} \\
\hline & \multicolumn{2}{|l|}{ Mean \pm SD } & \\
\hline Weight (kg) & $92.36 \pm 13.52$ & $73.52 \pm 12.31$ & $<0.0001 *$ \\
\hline BMI (Kg/m2) & $35.71 \pm 5.09$ & $28.64 \pm 5.35$ & $<0.0001 *$ \\
\hline Waist circumference (cm) & $115.42 \pm 12.30$ & $98.06 \pm 9.50$ & $<0.0001^{*}$ \\
\hline
\end{tabular}

*Significant

2- There was a significant variability between FBG in the NAFLD and the control group (Pvalue: 0.009), while there was no statistically significant association between duration of DM and the presence of NAFLD (P-value: 0.433). (table 2)

Table (2): comparison of DM among study groups

\begin{tabular}{|c|c|c|c|}
\hline \multirow[t]{2}{*}{ Variable } & $\begin{array}{l}\text { NAFLD group } \\
(\mathrm{N}=50)\end{array}$ & $\begin{array}{l}\text { Control group } \\
(\mathrm{N}=50)\end{array}$ & \multirow[t]{2}{*}{ P-value } \\
\hline & \multicolumn{2}{|l|}{ Mean \pm SD } & \\
\hline $\begin{array}{l}\text { Duration of DM } \\
\text { (years) }\end{array}$ & $3.6 \pm 3.4$ & $4.3 \pm 4.6$ & 0.433 \\
\hline FBG (mg/dl) & $168.20 \pm 61.19$ & $140.72 \pm 39.58$ & $0.009 *$ \\
\hline
\end{tabular}

*Significant 
3- This study showed that mean values of HOMA-IR and fasting insulin level are statistical significantly different between NAFLD and control groups (P-value: <0.0001). (table 3)

Table (3): Differences in fasting insulin and HOMA-IR according to study groups

\begin{tabular}{|c|c|c|c|}
\hline \multirow[t]{2}{*}{ Variable } & $\begin{array}{l}\text { Fatty liver group } \\
(\mathrm{N}=50)\end{array}$ & $\begin{array}{l}\text { No fatty liver group } \\
(\mathrm{N}=50)\end{array}$ & \multirow[t]{2}{*}{ P-value } \\
\hline & \multicolumn{2}{|l|}{ Mean \pm SD } & \\
\hline $\begin{array}{l}\text { Fasting insulin } \\
\text { (mg/dl) }\end{array}$ & $17.49 \pm 10.98$ & $9.89 \pm 7.31$ & $<0.0001 *$ \\
\hline HOMA-IR & $7.49 \pm 5.38$ & $3.41 \pm 2.69$ & $<0.0001 *$ \\
\hline
\end{tabular}

*Significant

4- ROC curve (Fig. 3) was done to estimate an appropriate cut-off value of HOMA-IR. The optimal threshold for the HOMA-IR value in discriminating NAFLD among T2DM patients was 4.21. The sensitivity, specificity, and total accuracy associated with a HOMA-IR cut-off value of 4.21 were $72 \%, 68 \%$ and $70 \%$, respectively (table 4 ). 


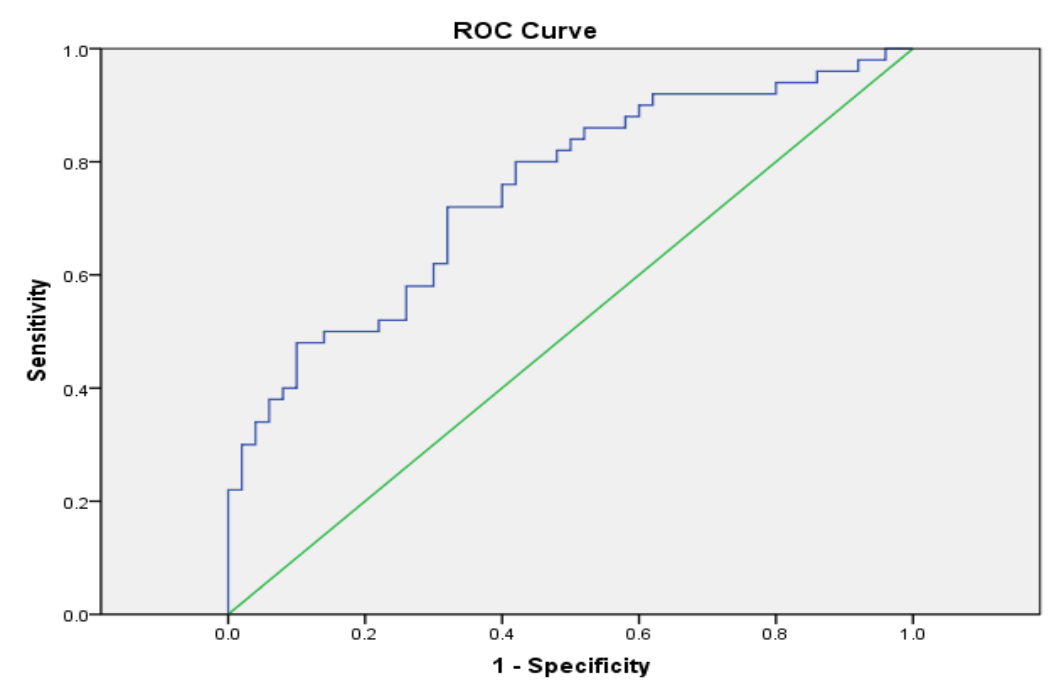

Figure (1): ROC curve of HOMA-IR for differentiating diabetic patients who have fatty liver from not have.

Table (4): Accuracy of HOMA-IR for differentiating diabetic patients who have NAFLD from not have

\begin{tabular}{|c|c|c|c|c|c|c|}
\hline & AUC & P-value & $\begin{array}{c}\text { Cut off } \\
\text { point }\end{array}$ & $\begin{array}{c}\text { Sensitivity } \\
(\boldsymbol{\%})\end{array}$ & $\begin{array}{c}\text { Specificity } \\
(\boldsymbol{\%})\end{array}$ & $\begin{array}{c}\text { Total } \\
\text { accuracy }\end{array}$ \\
\hline HOMA-IR & 0.748 & $\mathbf{< 0 . 0 0 0 1}^{*}$ & 4.21 & 72.0 & 68.0 & 70.0 \\
\hline
\end{tabular}

\section{Discussion}

Patients with type 2 diabetes mellitus (T2DM) appear to have an increased risk of developing NAFLD, with prevalence of 49.6 to $74 \%$ reported. Since the prevalence of T2DM has risen dramatically in recent years, NAFLD represents a major potential threat to public health.[6].
Invasive or expensive diagnostic options, such as liver biopsy or imaging tests, should be utilized in the context of available resources, with consideration of the burden on healthcare systems and the limitation of current effective treatments for NAFLD [6]. Accordingly, it would seem reasonable to explore the diagnostic potential of clinical factors as predictors of NAFLD in high-risk 
patients, in order to select patients for more invasive or expensive tests [6].

Several studies have shown an association between insulin resistance (IR) and NAFLD $[6,7]$. We aimed to evaluate the diagnostic accuracy of HOMA-IR for NAFLD in T2DM and sought to estimate an optimal cut-off value for discriminating NAFLD from non-NAFLD cases.

This study showed that there is statistically significant association between mean value of HOMA-IR and the presence of NAFLD (P-value: <0.0001). A HOMA-IR value of 4.2 showed the best sensitivity, specificity, and total accuracy values $(72 \%, 68 \%$ and $70 \%$, respectively). This was consistent with a recent study conducted by Gutierrez-Buey et al. that demonstrated an association between IR and NAFLD in T2DM adults, and suggested that HOMA-IR may be useful in discriminating NAFLD in these patients, also estimated a HOMA-IR cut-off value of 4.5 with sensitivity and specificity of $66 \%$ and $93 \%$, respectively [7].

In this study, diabetic patients with NAFLD had a higher FBG levels than that of diabetic patients without NAFLD (P-value: 0.009). This was similar to results of a study by Junker et al, that found higher FBG in
T2DM and NAFLD group than T2DM and normal liver group [8]. This was explained by decreased insulin sensitivity in patients with NAFLD.

In the present study, compared to nonNAFLD group, NAFLD group had a significant higher level of fasting insulin $(17.49 \pm 10.98$ Vs $9.89 \pm 7.31, \mathrm{P}=<0.0001)$. This was similar to a recent study, by Israt and Liaquat, conducted on 74 subjects with IGT (diagnosed by 75g OGTT), found significantly higher levels of fasting serum insulin (17.26 \pm 8.49 vs. $13.38 \pm 4.40$, $\mathrm{p}=0.015)$, postprandial serum insulin (90.06 \pm 42.23 vs. $69.87 \pm 38.10, \mathrm{p}=0.034)$ and HOMA-IR $(2.20 \pm 1.0$ vs. $1.73 \pm 0.55$, $\mathrm{p}=0.012$ ) in NAFLD group (diagnosed by U/S) than in non-NAFLD group [9].

Our study had several limitations. The diagnosis of NAFLD was based on ultrasonography and was not confirmed by liver biopsy. Correlation between the different stages of NAFLD (by the histologic picture) and the levels of insulin resistance could not be done.

Conclusion: HOMA-IR is independently associated with the presence of NAFLD in adults with T2DM, and might potentially be 
applied in clinical practice as a screen for

\section{References}

1-Younossi ZM, Koenig AB, Abdelatif D; et al (2016): Global epidemiology of nonalcoholic fatty liver disease-metaanalytic assessment of prevalence, incidence, and outcomes. Hepatology; 64(1):73-84.

2-Chalasani N, Younossi Z, Lavine JE; et al (2012): The diagnosis and management of non-alcoholic fatty liver disease: practice guideline by the American Gastroenterological Association, American Association for the Study of Liver Diseases, and American College of Gastroenterology. Gastroenterology; 142(7):1592-609.

3-Giorda C, Forlani G, Manti R, et al (2017): Occurrence over time and regression of nonalcoholic fatty liver disease in type 2 diabetes. Diabetes Metab Res Rev; 33:e2878 .

4-European Association for the Study of the Liver, European Association for the Study of Diabetes, European Association for the Study of Obesity (2016): EASL-EASDEASO Clinical Practice Guidelines for the
NAFLD in type 2 diabetic patients.

management of non-alcoholic fatty liver disease. Diabetologia; 59(6):1121-1140.

5-Cruz MAF, Cruz JF, Macena LB, et al (2015): Association of the Nonalcoholic Hepatic Steatosis and Its Degrees With the Values of Liver Enzymes and Homeostasis Model Assessment-Insulin Resistance Index. Gastroenterology Research; 8(5):260-264.

6-European Association for the Study of the Liver, European Association for the Study of Diabetes, European Association for the Study of Obesity (2016): EASL-EASDEASO Clinical Practice Guidelines for the management of non-alcoholic fatty liver disease. Diabetologia; 59(6):1121-1140

7-Gutierrez-Buey G, Núñez-Córdoba JM, Llavero-Valero M et al (2017): Is HOMAIR a potential screening test for nonalcoholic fatty liver disease in adults with type 2 diabetes? Eur J Intern Med; 41:74-78.

8-Junker AE, Gluud L, Holst JJ et al (2016): Diabetic and nondiabetic patients with nonalcoholic fatty liver disease have an impaired incretin effect and fasting hyperglucagonaemia. J Intern Med; 279(5):485-493. 
9-Israt AH and Liaquat A (2016): 10-Rhee E-J, Lee W-Y, et al (2011): Nonalcoholic Fatty Liver Disease and its Hyperinsulinemia and the Development of association with Insulin Resistance: A Study Nonalcoholic Fatty Liver Disease in from Bangladeshi Newly Diagnosed Nondiabetic Adults. The American Journal Impaired Glucose Tolerance Subjects. J of Medicine; 124 (1): 69- 76.

Diabetes Metab; 7:688. 